\title{
Simulations of $\mathrm{H}_{2}$ Sorption in an Anthracene-Functionalized rht-Metal-Organic Framework
}

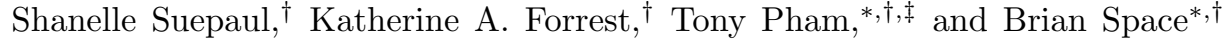 \\ ${ }^{\dagger}$ Department of Chemistry, University of South Florida, \\ 4202 East Fowler Avenue., CHE205, Tampa, Florida 33620-5250, United States \\ $\ddagger$ Department of Chemistry, Biochemistry, and Physics, The University of Tampa, \\ 401 West Kennedy Boulevard, Tampa, Florida 33606-1490, United States \\ *tpham4@mail.usf.edu; brian.b.space@gmail.com
}

\section{Electronic Structure Calculations}

The partial charges for the various chemically distinct atoms in MFM-132 (Figure S1) were determined through electronic structure calculations on different fragments that were extracted from the crystal structure of the MOF; selections were made using the Molden program. ${ }^{1}$ The 8 fragments that were considered for MFM-132 in this work are shown in Figure S2(a-h). The NWChem ab initio simulation software ${ }^{2}$ was used to calculate the electrostatic potential surface (ESP) of each fragment. For these calculations, all atoms were treated with the $6-31 \mathrm{G}^{*}$ basis set because it yields overpolarized charges that are more realistic for the crystalline environment. ${ }^{3}$ The CHELPG method ${ }^{4}$ was used to fit the charges onto the atomic centers of each fragment to reproduce the respective ESPs. The calculated average partial charges for each chemically distinct atom within the 8 considered fragments are provided in Table S3.

Excellent agreement was obtained for the partial charges for the unique atoms between the fragments, with standard deviations of no greater than $0.03 e^{-}$(see Table S3). The average partial charges for each of the chemically distinct atoms between the fragments was multiplied by the number of each type of atom within the unit cell to find the total charge on the unit cell. In doing this, the overall charge of the unit cell was found to be positive. Therefore, all atoms with positive charges were multiplied by a factor (absolute value of the ratio of the total negative charge to the total positive charge) to bring the magnitude of the total positive charge to be equivalent with that for the total negative charge. The resulting "scaled" partial charges for each chemically distinct atom in MFM-132 can be found in Table S3. These partial charges were used for the simulations in this work to calculate stationary electrostatic interactions.

Calculations were also performed on a $\left[\mathrm{Cu}_{2}\left(\mathrm{O}_{2} \mathrm{CH}\right)_{4}\right]$ cluster (Fragment 9, Figure S2(i)) taken from the crystal structure of MFM-132 as a control to confirm that the partial charge for the $\mathrm{Cu} 2$ ion is greater than that for the $\mathrm{Cu} 1$ ion even in the absence of the linkers. The averaged partial charges for the unique atoms in this fragment are displayed in Table S2. 


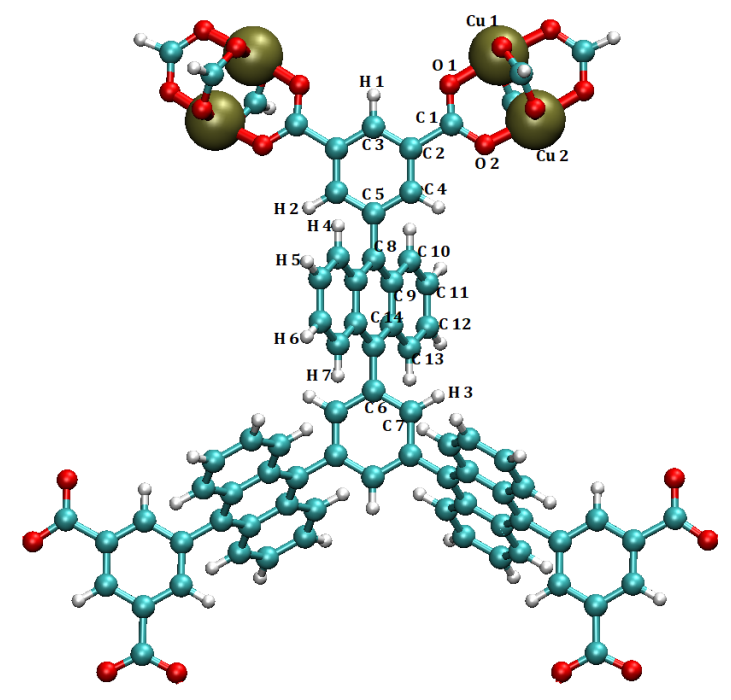

Figure S1. A gas phase fragment (Fragment 1, Figure S2(a)) of MFM-132 showing the numerical labels of the chemically distinct atoms in the MOF. Such labels correspond to Tables S1, S2, and S3. Atom colors: C = cyan, H = white, O = red, Cu = tan.

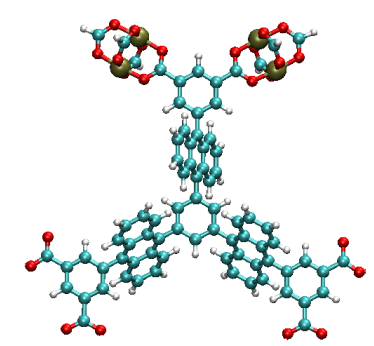

(a) Fragment 1

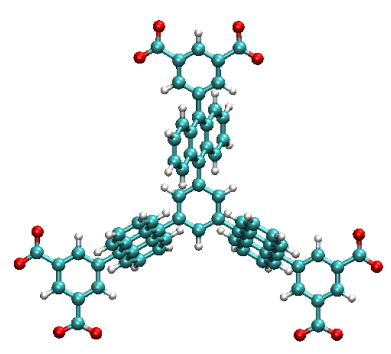

(e) Fragment 5

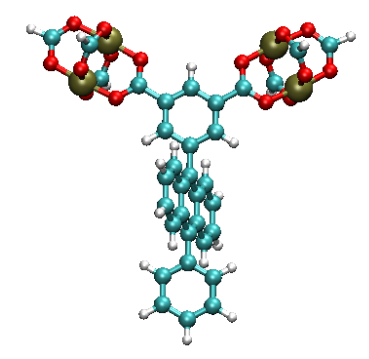

(b) Fragment 2

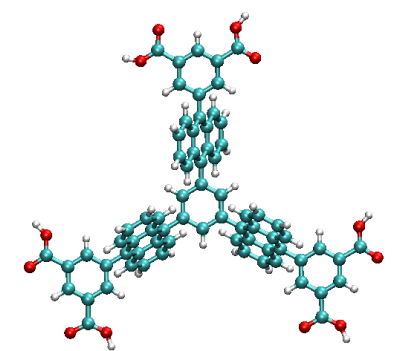

(f) Fragment 6

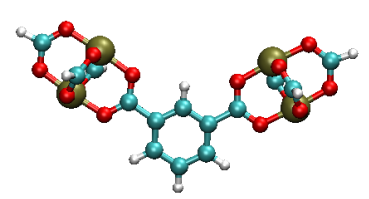

(c) Fragment 3

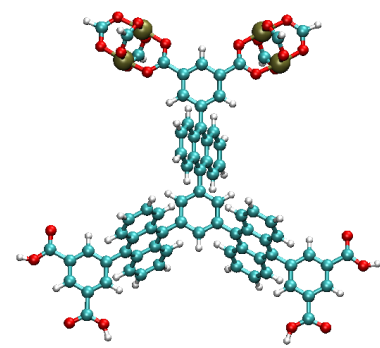

(g) Fragment 7

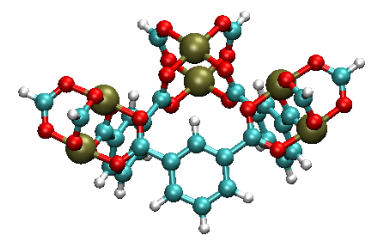

(d) Fragment 4

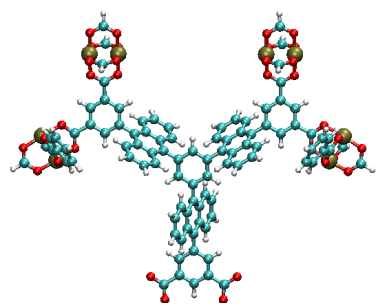

(h) Fragment 8

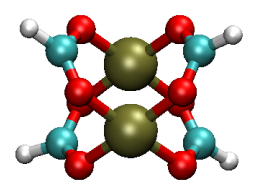

(i) Fragment 9

Figure S2. Gas phase fragments extracted from the crystal structure of MFM-132 that were used for the electronic structure calculations. Atom colors: $\mathrm{C}=$ cyan, $\mathrm{H}=$ white, $\mathrm{O}=$ red, $\mathrm{Cu}=$ tan. 
Table S1. Calculated average partial charges $\left(e^{-}\right)$for the various chemically distinct atoms for the 8 considered fragments that were selected for MFM-132. Atomic site numbering corresponds to Figure S1, while label of fragments correspond to Figure S2.

\begin{tabular}{|c|c|c|c|c|c|c|c|c|}
\hline Atom Label & Fragment 1 & Fragment 2 & Fragment 3 & Fragment 4 & Fragment 5 & Fragment 6 & Fragment 7 & Fragment 8 \\
\hline $\mathrm{Cu} 1$ & 1.1034 & 1.1097 & 1.1085 & 1.1033 & - & - & 1.0745 & 1.0928 \\
\hline $\mathrm{Cu} 2$ & 1.6100 & 1.5711 & 1.5937 & 1.5753 & - & - & 1.5805 & 1.6055 \\
\hline C 1 & 0.9427 & 0.9616 & - & - & 0.8944 & 0.8842 & 0.9628 & 0.9423 \\
\hline C 2 & -0.2071 & -0.2058 & - & - & - & - & -0.2040 & -0.2296 \\
\hline C 3 & -0.0835 & - & - & -0.1090 & -0.0988 & - & - & -0.1136 \\
\hline C 4 & -0.1551 & -0.1428 & - & - & - & - & -0.1310 & 0.1335 \\
\hline C 5 & 0.4206 & 0.3548 & - & - & 0.2780 & 0.3183 & 0.3408 & - \\
\hline C 6 & 0.3660 & - & - & - & 0.4413 & 0.4075 & - & 0.4033 \\
\hline C 7 & -0.4274 & - & - & - & -0.4378 & -0.4481 & - & -0.4402 \\
\hline C 8 & - & -0.3524 & - & - & - & -0.3059 & -0.3410 & -0.2959 \\
\hline C 9 & 0.2451 & 0.2054 & - & - & - & - & 0.2046 & - \\
\hline C 10 & -0.2206 & -0.2271 & - & - & - & -0.2062 & -0.2138 & -0.2211 \\
\hline C 11 & - & -0.1296 & - & - & -0.1401 & - & -0.1209 & -0.1332 \\
\hline C 12 & - & - & - & - & -0.1608 & -0.1751 & -0.1616 & -0.1771 \\
\hline C 13 & - & - & - & - & -0.1626 & -0.1665 & -0.1681 & -0.1698 \\
\hline C 14 & - & - & - & - & -0.0222 & -0.0362 & -0.0178 & -0.0250 \\
\hline C 15 & - & - & - & - & -0.0952 & -0.0716 & -0.0109 & - \\
\hline O 1 & -0.7893 & -0.7950 & -0.7545 & -0.7301 & - & - & -0.7957 & -0.7742 \\
\hline O 2 & -0.7511 & -0.7590 & -0.7400 & -0.7237 & - & - & -0.7538 & -0.7603 \\
\hline H 1 & 0.1792 & 0.1953 & - & 0.1878 & - & - & 0.1961 & 0.1882 \\
\hline H 2 & 0.1580 & 0.1524 & - & - & - & - & 0.1442 & 0.1506 \\
\hline H 3 & 0.1543 & - & - & - & 0.1662 & 0.1568 & - & 0.1601 \\
\hline H 4 & 0.1344 & 0.1365 & - & - & 0.1207 & - & 0.1372 & 0.1342 \\
\hline H 5 & - & - & 0.1358 & - & - & 0.1226 & 0.1298 & 0.1359 \\
\hline H 6 & 0.1406 & - & - & - & - & 0.1300 & 0.1261 & 0.1315 \\
\hline H 7 & 0.1188 & - & - & - & - & 0.1252 & 0.1224 & 0.1198 \\
\hline
\end{tabular}

Table S2. Calculated average partial charges $\left(e^{-}\right)$for the $\mathrm{Cu} 1, \mathrm{Cu} 2, \mathrm{O} 1$, and $\mathrm{O} 2$ atoms for the $\left[\mathrm{Cu}_{2}\left(\mathrm{O}_{2} \mathrm{CH}\right)_{4}\right]$ cluster $(\mathrm{Fragment}$ 9, Figure S2(i)) from MFM-132 that was used as a control. Atomic site numbering corresponds to Figure S1.

\begin{tabular}{|c|c|}
\hline Atom Label & $q\left(e^{-}\right)$ \\
\hline Cu 1 & 1.1419 \\
\hline Cu 2 & 1.6267 \\
\hline O 1 & -0.7816 \\
\hline O 2 & -0.7726 \\
\hline
\end{tabular}


Table S3. Calculated average and standard deviation (STD) of the partial charges $\left(e^{-}\right)$for the various chemically distinct atoms between the 8 considered fragments for MFM-132. The number of each type of atom within a unit cell of the MOF is also included. In order to make the overall charge of the system neutral, it was necessary to multiply all averaged positive charges by 0.998047 ; all averaged negative charges remained unchanged. The "scaled" partial charges were used for the simulations in this work. Atomic site numbering corresponds to Figure S1.

\begin{tabular}{|c|c|c|c|c|}
\hline Atom Label & \# in Unit Cell & Average & STD & Scaled \\
\hline Cu 1 & 48 & 1.0924 & 0.0298 & $\mathbf{1 . 0 9 0 3}$ \\
\hline C 2 & 48 & 1.5893 & 0.0162 & $\mathbf{1 . 5 8 6 1}$ \\
\hline C 1 & 192 & 0.9313 & 0.0339 & $\mathbf{0 . 9 2 9 5}$ \\
\hline C 2 & 192 & -0.2116 & 0.0120 & $-\mathbf{0 . 2 1 1 6}$ \\
\hline C 3 & 96 & -0.1071 & 0.0075 & $\mathbf{- 0 . 1 0 7 1}$ \\
\hline C 4 & 192 & -0.1406 & 0.0109 & $\mathbf{- 0 . 1 4 0 6}$ \\
\hline C 5 & 96 & 0.3755 & 0.0355 & $\mathbf{0 . 3 7 4 8}$ \\
\hline C 6 & 96 & 0.4045 & 0.0308 & $\mathbf{0 . 4 0 3 7}$ \\
\hline C 7 & 96 & -0.4384 & 0.0085 & $\mathbf{- 0 . 4 3 8 4}$ \\
\hline C 8 & 96 & -0.3238 & 0.0272 & $\mathbf{- 0 . 3 2 3 8}$ \\
\hline C 9 & 192 & 0.2184 & 0.0231 & $\mathbf{0 . 2 1 7 9}$ \\
\hline C 10 & 192 & -0.2177 & 0.0080 & $\mathbf{- 0 . 2 1 7 7}$ \\
\hline C 11 & 192 & -0.1310 & 0.0080 & $\mathbf{- 0 . 1 3 1 0}$ \\
\hline C 12 & 192 & -0.1686 & 0.0087 & $\mathbf{- 0 . 1 6 8 6}$ \\
\hline C 13 & 192 & -0.1667 & 0.0031 & $\mathbf{- 0 . 1 6 6 7}$ \\
\hline C 14 & 192 & -0.0253 & 0.0078 & $-\mathbf{0 . 0 2 5 3}$ \\
\hline C 15 & 96 & -0.0592 & 0.0435 & $-\mathbf{0 . 0 5 9 2}$ \\
\hline O 1 & 192 & -0.7731 & 0.0263 & $\mathbf{- 0 . 7 7 3 1}$ \\
\hline O 2 & 192 & -0.7480 & 0.0139 & $\mathbf{- 0 . 7 4 8 0}$ \\
\hline H 1 & 96 & 0.1893 & 0.0069 & $\mathbf{0 . 1 8 9 0}$ \\
\hline H 2 & 192 & 0.1513 & 0.0057 & $\mathbf{0 . 1 5 1 0}$ \\
\hline H 3 & 96 & 0.1593 & 0.0051 & $\mathbf{0 . 1 5 9 0}$ \\
\hline H 4 & 192 & 0.1326 & 0.0068 & $\mathbf{0 . 1 3 2 3}$ \\
\hline H 5 & 192 & 0.1310 & 0.0063 & $\mathbf{0 . 1 3 0 7}$ \\
\hline H 6 & 192 & 0.1320 & 0.0061 & $\mathbf{0 . 1 3 1 8}$ \\
\hline H 7 & 192 & 0.1215 & 0.0029 & $\mathbf{0 . 1 2 1 3}$ \\
\hline & & & & \\
\hline
\end{tabular}




\section{$\mathbf{H}_{2}$ Potentials}

Table S4. Force field parameters for the $\mathrm{H}_{2}$ potentials ${ }^{5,6}$ used in this work. COM refers to the center-of-mass site and OS refers to the off-site position.

\begin{tabular}{|c|c|c|c|c|c|c|}
\hline Model & Site & $r(\AA)$ & $\epsilon(\mathrm{K})$ & $\sigma(\AA)$ & $q\left(e^{-}\right)$ & $\alpha^{\circ}\left(\AA^{3}\right)$ \\
\hline Buch & $\mathrm{COM}$ & 0.00000 & 34.20000 & 2.96000 & 0.00000 & 0.00000 \\
\hline \multirow{3}{*}{ BSS } & $\mathrm{COM}$ & 0.00000 & 8.85160 & 3.22930 & -0.74640 & 0.00000 \\
& $\mathrm{H}$ & 0.37100 & 0.00000 & 0.00000 & 0.37320 & 0.00000 \\
& OS & 0.32900 & 4.06590 & 2.34060 & 0.00000 & 0.00000 \\
\hline \multirow{4}{*}{ BSSP } & $\mathrm{COM}$ & 0.00000 & 12.76532 & 3.15528 & -0.74640 & 0.69380 \\
& $\mathrm{H}$ & 0.37100 & 0.00000 & 0.00000 & 0.37320 & 0.00044 \\
& OS & 0.36300 & 2.16726 & 2.37031 & 0.00000 & 0.00000 \\
\hline
\end{tabular}




\section{Calculation of Thermodynamic Quantities}

The average number of $\mathrm{H}_{2}$ molecules sorbed in MFM-132 $(\langle N\rangle)$ from grand canonical Monte Carlo (GCMC) simulations was calculated using the following expression that is based on the grand canonical ensemble: ${ }^{7}$

$$
\langle N\rangle=\frac{1}{\Xi} \sum_{N=0}^{\infty} e^{\beta \mu N}\left\{\prod_{i=1}^{3 N} \int_{\infty}^{-\infty} d x_{i}\right\} N e^{-\beta U_{F H}\left(x_{i}, \ldots, x_{3 N}\right)}
$$

where $\Xi$ is the grand canonical partition function, $\beta$ is the inverse of the Boltzmann constant $(k)$ multiplied by the temperature $(T), \mu$ is the chemical potential of the gas reservoir, and $U_{F H}$ is the total potential energy of the MOF- $\mathrm{H}_{2}$ system that accounts for Feynman-Hibbs quantum corrections. ${ }^{8}$ Once $\langle N\rangle$ was calculated at a given state point, it was converted to units of weight percent (wt \%) for comparison to experimental data. This quantity was calculated by the following:

$$
w t \%=\frac{\langle N\rangle m}{M+\langle N\rangle m} \times 100 \%
$$

where $m$ is the molar mass of $\mathrm{H}_{2}$ and $M$ is the molar mass of the unit cell of MFM-132.

$\mu$ for $\mathrm{H}_{2}$ was determined for a range of temperatures and pressures through the thermodynamic BACK equation of state. ${ }^{9}$ Feynman-Hibbs quantum corrections to the fourth order were applied to the simulations at all state points considered herein to treat energetically dominant repulsion/dispersion interactions for $\mathrm{H}_{2}$ sorption at cryogenic temperatures. ${ }^{10,11}$ The expression for $U_{F H}$ is the following: ${ }^{8}$

$$
U_{F H}=U+\frac{\beta \hbar^{2}}{24 \mu_{m}}\left(U^{\prime \prime}+\frac{2}{r} U^{\prime}\right)+\frac{\beta^{2} \hbar^{4}}{1152 \mu_{m}^{2}}\left(\frac{15}{r^{3}} U^{\prime}+\frac{4}{r} U^{\prime \prime \prime}+U^{\prime \prime \prime \prime}\right)
$$

where $U$ is the (uncorrected) total potential energy of the MOF- $\mathrm{H}_{2}$ system, $\hbar$ is the reduced Planck's constant, $\mu_{m}$ is the reduced mass for $\mathrm{H}_{2}$, and the primes are the derivatives with respect to pair separation $r$. $U$ was calculated through the following analytical functional form:

$$
U=U_{r d}+U_{e s}+U_{p o l}
$$

where $U_{r d}$ is the repulsion/dispersion energy calculated using the Lennard-Jones $12-6$ potential ${ }^{12}$ with Lorentz-Bertholet mixed $\epsilon$ and $\sigma$ parameters, ${ }^{13} U_{e s}$ is the permanent electrostatic energy calculated using Ewald summation of the partial charges ${ }^{14}$ and $U_{p o l}$ is the many-body polarization energy, which was calculated explicitly using a Thole-Applequist type model. ${ }^{15-18}$ For simulations using the BSS model, $U_{\text {pol }}$ was equal to 0 , while both $U_{e s}$ and $U_{\text {pol }}$ were equal to 0 for simulations using the Buch model.

In GCMC, the isosteric heat of adsorption $\left(Q_{s t}\right)$ values were calculated using fluctuations in $N$ and $U$ through the following statistical mechanical expression: ${ }^{19}$

$$
Q_{s t}=-\frac{\langle N U\rangle-\langle N\rangle\langle U\rangle}{\left\langle N^{2}\right\rangle-\langle N\rangle^{2}}+k T
$$

where $\langle\ldots\rangle$ indicates the ensemble average. This is in contrast to the experimental method of obtaining the $Q_{s t}$ values, which are typically determined from numerical analysis of at least two sorption isotherms at different temperatures, followed by interpolation over a range of uptakes using the Clausius-Clapeyron equation. ${ }^{20}$ 


\section{Binding Energy Calculations}

Ab initio calculations at the Hartree-Fock ( $\mathrm{HF}$ ) level of theory were carried out to determine the binding energy for $\mathrm{H}_{2}$ sorbed in (1) the binding pocket created by the partial overlap of the anthracenyl rings from three neighboring linkers and (2) at the $\mathrm{Cu} 2$ open-metal site in MFM-132 using NWChem. ${ }^{2}$ This was done using the output from the GCMC simulations by extracting a chemically sensible fragment with a $\mathrm{H}_{2}$ molecule sorbed at each site. The considered fragment containing $\mathrm{H}_{2}$ sorbed at both binding sites are shown in Figure S3. For each site, the position of the $\mathrm{H}_{2}$ molecule was first optimized within the selected fragment. Afterward, the binding energy was calculated by subtracting the sum of the energy of the fragment itself and that of the bare $\mathrm{H}_{2}$ molecule from the total energy of the fragment with the sorbed $\mathrm{H}_{2}$. All atoms were treated with the 6-31G* basis set for these calculations. The raw results from the binding energy calculations for $\mathrm{H}_{2}$ sorbed at both sites are shown in Table S5.

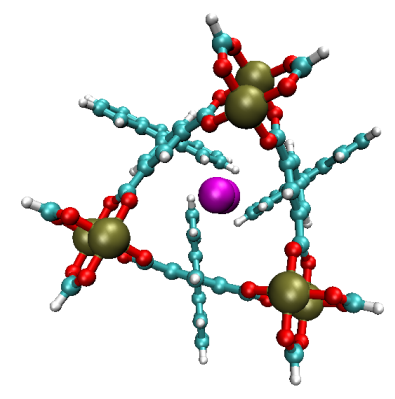

(a) Binding Pocket

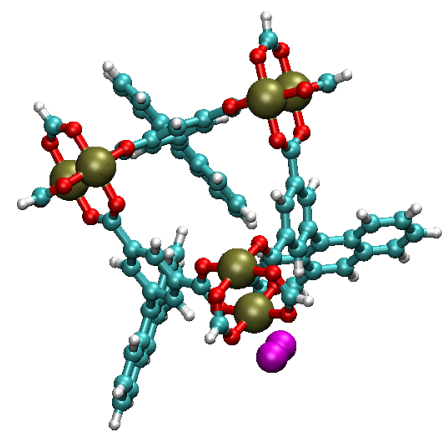

(b) $\mathrm{Cu} 2$

Figure S3. Fragment extracted from the crystal structure of MFM-132 containing a $\mathrm{H}_{2}$ molecule sorbed at (a) the binding pocket and (b) $\mathrm{Cu} 2$ open-metal site that were used in calculations of the binding energy. Atom colors: $\mathrm{C}=\mathrm{cyan}, \mathrm{H}=\mathrm{white}, \mathrm{O}=\mathrm{red}, \mathrm{Cu}=$ tan.

Table S5. Energies obtained from HF calculations for $\mathrm{H}_{2}$ sorbed at the binding pocket (Figure S3(a)) and Cu 2 (Figure S3(b)) sites in MFM-132 using NWChem. ${ }^{2}$

\begin{tabular}{|c|c|c|}
\hline Component & Binding Pocket & $\mathrm{Cu} 2$ \\
\hline Fragment $+\mathrm{H}_{2}$ (Hartree) & -14380.8192 & -14380.8187 \\
\hline Fragment only (Hartree) & -14379.6901 & -14379.6901 \\
\hline $\mathrm{H}_{2}$ only (Hartree) & -1.1268 & -1.1268 \\
\hline Binding energy (Hartree) & -0.0023 & -0.0018 \\
\hline Binding energy $\left(\mathrm{kJ} \mathrm{mol}^{-1}\right)$ & -6.0386 & -4.7259 \\
\hline
\end{tabular}




\section{References}

${ }^{1}$ Schaftenaar, G.; Noordik, J. H. J. Comput. Aided Mol. Des. 2000, 14, 123-134.

${ }^{2}$ Valiev, M.; Bylaska, E.; Govind, N.; Kowalski, K.; Straatsma, T.; Dam, H. V.; Wang, D.; Nieplocha, J.; Apra, E.; Windus, T.; et al. Comput. Phys. Commun. 2010, 181, 1477-1489.

${ }^{3}$ Cornell, W. D.; Cieplak, P.; Bayly, C. I.; Gould, I. R.; Merz, K. M.; Ferguson, D. M.; Spellmeyer, D. C.; Fox, T.; Caldwell, J. W.; Kollman, P. A. J. Am. Chem. Soc. 1995, 117, 5179-5197.

${ }^{4}$ Breneman, C. M.; Wiberg, K. B. J. Comput. Chem. 1990, 11, 361-373.

${ }^{5}$ Buch, V. J. Chem. Phys. 1994, 100, 7610-7629.

${ }^{6}$ Belof, J. L.; Stern, A. C.; Space, B. J. Chem. Theory Comput. 2008, 4, 1332-1337.

7 Frenkel, D.; Smit, B. Understanding Molecular Simulation: From Algorithms to Applications; Academic Press: New York, 2002; pp. 129.

8 Sesé, L. M. Mol. Phys. 1993, 78, 1167-1177.

9 Boublík, T. Fluid Phase Equilib. 2006, 240, 96-100.

10 Garberoglio, G.; Skoulidas, A. I.; Johnson, J. K. J. Phys. Chem. B 2005, 109, 13094-13103.

11 Liu, J.; Culp, J. T.; Natesakhawat, S.; Bockrath, B. C.; Zande, B.; Sankar, S. G.; Garberoglio, G.; Johnson, J. K. J. Phys. Chem. $C$ 2007, 111, 9305-9313.

12 Jones, J. Proc. R. Lond. Soc. A 1924, 106, 463-477.

13 Allen, M. P.; Tildesley, D. J. Computer Simulation of Liquids; Oxford University Press: Oxford, United Kingdom, 1989 ; pp. $126-131$.

14 Yeh, I.-C.; Berkowitz, M. L. J. Chem. Phys. 1999, 111, 3155-3162.

15 Applequist, J.; Carl, J. R.; Fung, K.-K. J. Am. Chem. Soc. 1972, 94, 2952-2960.

16 Thole, B. T. Chem. Phys. 1981, 59, 341-350.

17 Van Duijnen, P. T.; Swart, M. J. Phys. Chem. A 1998, 102, 2399-2407.

18 McLaughlin, K.; Cioce, C. R.; Pham, T.; Belof, J. L.; Space, B. J. Chem. Phys. 2013, 139, 184112.

19 Nicholson, D.; Parsonage, N. G. Computer Simulation and the Statistical Mechanics of Adsorption; Academic Press: London, 1982; pp. 97.

20 Pan, H.; Ritter, J. A.; Balbuena, P. B. Langmuir 1998, 14, 6323-6327. 